\title{
Time Evolution of Gibbs States for an Anharmonic Lattice
}

\author{
C. Marchioro ${ }^{1 \star}$, A. Pellegrinotti ${ }^{1 \star}$, M. Pulvirenti ${ }^{2 \star}$, and Yu. Suhov ${ }^{1 \star \star}$ \\ 1 Istituto Matematico, Università di Camerino, I-62032 Camerino (MC), Italy \\ ${ }^{2}$ Istituto Matematico, Università dell 'Aquila, I-l'Aquila, Italy, and \\ Istituto Matematico, Università di Roma, I-Roma, Italy
}

\begin{abstract}
In this paper we study the time evolution of a regular class of states of an infinite classical system of anharmonic oscillators. The conditional probabilities are investigated and an explicit form for these is given.
\end{abstract}

\section{Introduction}

One of the main problem in non equilibrium Statistical Mechanics is to study the time evolution of states (i.e. probability measures on the phase space) of infinite interacting classical systems. A natural way is to consider the time evolution as described by a flow on the phase space arising from the Newton law of the motion.

The problem of constructing such a flow was solved in a satisfactory way for some classes of particle systems in [1], [2] and for anharmonic oscillators in [3]. Other results which are specifically related to the equilibrium situation were obtained in [4-8].

The next step is to study the time evolution of states, implemented by the flow on the phase space. An approach proposed in [9] and [10] is based on the hypothesis that a class of physically interesting states, the Gibbs states with respect to some Hamiltonian is preserved in the course of the evolution (the equilibrium states are precisely those states which are Gibbs with respect to the Hamiltonian governing the motion). The main advantage of this approach is that the change in time of the Hamiltonian of a given Gibbs state is described in a simple way, directly referred to finite-volume dynamics.

Such an approach was studied in [9] in the case of one dimensional hard core system interacting via a two body, bounded, short range potential. One of the main points in [9] is the use of the cluster dynamics that, roughly speaking, says that such systems behave in time as if they were formed by non interacting groups consisting of a finite number of particles.

* Research partially supported by Italian National Council of Research

$\star \star$ Permanent address: Institute for Problems of Information Transmission, USSR Academy of Science, Moscow, USSR

Research supported by Italian National Council of Research 
In this paper we study the time evolution of Gibbs states for a special class of anharmonic lattices in which the self-energy dominates over the interacting part. The interest of the anharmonic systems in the solid state physics is well known (see e.g. [11]). The dynamical flow we study here was investigated in [3]. Even if the dynamics of such systems is not, obviously, of cluster type, nevertheless it exhibits for many respects a simpler behaviour than the dynamics of continuous particle systems.

The plan of this work will be the following: in Sect. 2 we introduce the notations and formulate the results; in Sect. 3 the proofs are given; the Appendix is devoted in underlining some dynamical property we need.

\section{Notation and Result}

We consider the system of anharmonic oscillators on the $v$-dimensional cubic lattice $\mathbb{Z}^{v}$. The phase space of a single oscillator is assumed to be $\mathbb{R}^{1} \times \mathbb{R}^{1}$.

Definition 2.1. The phase space of the system under consideration is $\mathfrak{X}=\left\{\left(p_{i} q_{i}\right)_{i \in \mathbb{Z}^{v}} \mid q_{i} \in \mathbb{R}^{1}, p_{i} \in \mathbb{R}^{1}\right\}$. Points of $\mathfrak{X}$ are denoted $x, y$, etc. For every $\Lambda \subset \mathbb{Z}^{v}, \mathfrak{X}_{\Lambda}$ denotes the phase associated with the region $\Lambda ; \mathfrak{X}_{\Lambda}=\left\{\left(p_{i}, q_{i}\right)_{i \in \Lambda} \mid q_{i} \in \mathbb{R}^{1}, p_{i} \in \mathbb{R}^{1}\right\}$. Points of $\mathfrak{X}_{A}$ will be denoted by $x_{A}, y_{A}$, etc. The space $\mathfrak{X}$ and $\mathfrak{X}_{A}$ are equipped with the natural Tychonov topologies. The corresponding Borel $\sigma$-algebras are denoted by $\mathscr{B}$ and $\mathscr{B}_{\Lambda}$ and are the $\sigma$-algebras generated by the variables $\left(p_{i}, q_{i}\right)_{i \in \mathbb{Z}^{v}}$ and $\left(p_{i}, q_{i}\right)_{i \in \Lambda}$.

Definition 2.2. A state $\mu$ of the system of oscillators on $\mathbb{Z}^{v}$ is a probability measure on $\mathscr{B}$. Since $\mathfrak{X}$ is a Polish space, $\left(\mathfrak{X}, \mathscr{B}^{*}, \mu\right)$, where $\mathscr{B}^{*}$ is the completion of $\mathscr{B}$ w.t.r. $\mu$. is a Lebesgue space. [12].

Given a partition $\xi$ of $\mathfrak{X}$, we say that $\xi$ is measurable if there exists a countable family $\left\{f_{1} \ldots f_{n} \ldots\right\}$ of measurable functions, such that every atom $a_{\alpha}$ of the partition $\xi$ is labelled by $\alpha \in \mathbb{R}^{\mathbb{N}}$, and $a_{\alpha}=\left\{x \in \mathfrak{X} \mid f_{n}(x)=\alpha_{n} \ldots \alpha \equiv\left(\alpha_{i} \ldots \alpha_{n} \ldots\right)\right\}$.

Given a measurable partition $\xi$, let us consider the factor space $\mathfrak{X} / \xi$ whose points are the atoms of the partition. The canonical map $\Pi: \mathfrak{X} \rightarrow \mathfrak{X} / \xi$ which associates at every point of $\mathfrak{X}$ its atom, determines the measurable sets on $\mathfrak{X} / \xi$ as the sets whose inverse image is measurable in $\mathfrak{X}$.

Let $\mu_{\xi}$ be the measure on $\mathfrak{X} / \xi$ defined by $\mu_{\xi}(B)=\mu\left(\Pi^{-1}(B)\right)$. There exists a family $\{\mu(\cdot \mid a), a \in \xi\}$ of a measures on atoms $a \in \xi$ such that each $(a, \mu(\cdot \mid a))$ is a Lebesgue space. Furthermore, for all $A \in \mathscr{B}^{*}, A \cap a$ is $\mu(\cdot \mid a)$-measurable for $\mu_{\xi}$-a.a. $a \in \xi$ and :

$$
\mu(A)=\int_{\mathfrak{X} / \zeta} \mu_{\xi}(d a) \mu(A \cap a \mid a) .
$$

Such a family $\{\mu(\cdot \mid a)\}$ is unique mod 0 (see [12]) and is called the system of conditional probabilities of the state $\mu$ w.r.t. $\xi$.

In the sequel we shall use the following property of the conditional probabilities.

Let us consider two partitions, $\xi$ whose atoms we denote by $a$, and $\eta$ whose atoms we denote by $b$. Assume that $\eta$ is a refinement of $\xi$ that means that every $b$ is 
contained in some $a$. Then denoting by $a(x)$ (or $b(x))$ the atom of type $a$ (or $b$ ) which contains the point $x \in \mathfrak{X}$, it is not hard to prove that for $\mu$-a.a. $x \in \mathfrak{X}$, the following equality holds :

$$
\mu(A \mid a(x))=\int_{a(x)} \mu(A \mid b(y)) \mu(d y \mid a(x)) .
$$

Let us fix a bounded region $\Lambda$, and consider the following atoms

$$
a_{\Lambda}(x)=\left\{y \in \mathfrak{X} \mid(x)_{\Lambda^{c}}=(y)_{\Lambda^{c}}\right\} .
$$

Here $(x)_{\Omega}$ denote the restriction of $x$ to $\mathfrak{X}_{\Omega}, \Omega \subset \mathbb{Z}^{v}$. Let us consider $\xi_{\Lambda}$, the partition given by the atoms $a_{\Lambda}(x)$. $\xi$ is obviously measurable. Every atom $a_{A}(x)$ may be thought as $\mathfrak{X}_{\Lambda}$, hence $\mu\left(\cdot \mid a_{\Lambda}(x)\right)$ induces a measure on $\mathfrak{X}_{\Lambda}$ still denoted

$$
\mu\left(d x_{\Lambda} \mid a_{\Lambda}(x)\right)=\mu\left(d x_{\Lambda} \mid(x)_{\Lambda^{c}}\right) .
$$

Then for any bounded measurable function $f: \mathfrak{X} \rightarrow \mathbb{R}(2.1)$ reads as

$$
\int \mu(d x) f(x)=\int_{\mathfrak{X}} \mu(d x) \int_{\mathfrak{X}_{\Lambda}} \mu\left(d x_{\Lambda} \mid(x)_{\Lambda^{c}}\right) f\left(x_{\Lambda} \cup(x)_{\Lambda^{c}}\right) .
$$

Definition 2.3. Let $h$ be a real valued function on the set $\mathfrak{X}^{(0)}=\bigcup_{\Lambda \subset \mathbb{Z}^{v}} \mathfrak{X}_{\Lambda}$ ( $\Lambda$ finite) such that $\left.h\right|_{\mathfrak{x}_{\Lambda}}$ is $\mathscr{B}$-measurable for all finite $\Lambda \subset \mathbb{Z}^{v}$. Given finite subsets $\Lambda, \Lambda^{\prime} \subset \mathbb{Z}^{v}$, $\Lambda \cap \Lambda^{\prime}=\Phi$ and a pair $\left(x_{\Lambda}, x_{\Lambda^{\prime}}\right), x_{\Lambda} \in \mathfrak{X}_{\Lambda}, x_{\Lambda^{\prime}} \in \mathfrak{X}_{\Lambda^{\prime}}$, we set

$$
h\left(x_{\Lambda} \mid x_{\Lambda^{\prime}}^{\prime}\right)=h\left(x_{\Lambda} \cup x_{\Lambda^{\prime}}^{\prime}\right)-h\left(x_{\Lambda}\right)-h\left(x_{\Lambda^{\prime}}^{\prime}\right) \text {. }
$$

We say that $\mu$ is a Gibbs state corresponding to the generating function $h$, if for any finite $\Lambda \subset \mathbb{Z}^{v}$

i) the limit

$$
h\left(x_{\Lambda} \mid(x)_{\Lambda^{c}}\right)=\lim _{n \rightarrow \infty} h\left(x_{\Lambda} \mid(x)_{\Lambda n \backslash \Lambda}\right), \quad x_{\Lambda} \in \mathfrak{X}_{\Lambda}, \quad x \in \mathfrak{X}
$$

exists in the sense of convergence in measure $(\lambda \times \mu)$ over the cartesian product $\mathfrak{X}_{\Lambda} \times \mathfrak{X}$, where $\Lambda_{n}$ is the cube $[-n, n]^{v}, n \in \mathbb{N}$, and $\lambda$ denotes the Lebesgue measure on $\mathfrak{X}_{\Lambda}$;

ii) the integral

$$
\Xi_{\Lambda}(h ; x)=\int_{\mathfrak{x}_{\Lambda}} d \lambda\left(x_{\Lambda}\right) \exp \left[-h\left(x_{\Lambda}\right)-h\left(x_{\Lambda} \mid(x)_{\Lambda^{c}}\right)\right]
$$

is finite for $\mu-$ a.a. $x \in \mathfrak{X}$ and any finite $\Lambda \subset \mathbb{Z}^{\nu}$;

iii) For any $\Lambda \subset \mathbb{Z}^{v}$ the conditional probability system for $\mu$ w.r.t. $\xi_{\Lambda}$ is given by

$$
\mu\left(d x_{\Lambda} \mid(x)_{\Lambda^{c}}\right)=\frac{d \lambda\left(x_{\Lambda}\right)}{\Xi_{\Lambda}(h ; x)} \exp \left[-h\left(x_{\Lambda}\right)-h\left(x_{\Lambda} \mid(x)_{\Lambda^{c}}\right)\right] .
$$

Definition 2.4. The function $H: \mathfrak{X}^{(0)} \rightarrow \mathbb{R}^{1}$ defined by

$$
H\left(x_{\Lambda}\right)=\sum_{i \in \Lambda}\left(p_{i}^{2} / 2+k q_{i}^{2}+\lambda q_{i}^{4}-J q_{i} \sum_{j \in \Lambda \cap v_{2}} q_{d}\right)
$$

where $k, \lambda>0, \quad J \in \mathbb{R}^{1}, \quad v_{i}=\left\{j \in \mathbb{Z}^{v}|| i-j \mid=1\right\}, \quad|i-j|=\sum_{\alpha=1}^{v}\left|i_{\alpha}-j_{\alpha}\right|$ is called the 
Hamiltonian. The Hamiltonian (2.8) describes a physical model of anharmonic oscillators (with unitary mass). We choose a particular Hamiltonian for the sake of simplicity, but all our considerations are straightforwardly valid in the case of all Hamiltonians where the self-energy dominates the interacting part (see [3]).

Definition 2.5. By $\left\{\tilde{S}_{t}, t \in \mathbb{R}^{1}\right\}$ we denote the group of transformations $\mathfrak{X}^{(0)} \rightarrow \mathfrak{X}^{(0)}$ representing the motion of a finite system of oscillators with Hamiltonian $H$. Clearly, $\tilde{S}_{t} \mathfrak{X}_{\Lambda}=\mathfrak{X}_{\Lambda}$ for every $t$ and finite $\Lambda \subset \mathbb{Z}^{v}$.

Definition 2.6. For every $n \in \mathbb{N}$ put $\Lambda_{n}=[-n, n]^{v}$ and denote by $\left\{S_{t}^{(n)}, t \in \mathbb{R}^{1}\right\}$ the following group of transformation $\mathfrak{X} \rightarrow \mathfrak{X}$. Given $x \in \mathfrak{X},\left(S_{t}^{(n)} x\right)_{\Lambda_{n}^{c}}=x_{\Lambda_{n}^{c}}$ and $\left(S_{t}^{(n)} x\right)_{\Lambda_{n}}$ represents the solution of the Hamilton equations for the oscillators in $\Lambda_{n}$ interacting via the Hamiltonian $H$ and moving in the external field generated by the frozen oscillators outside $\Lambda_{n}$.

Definition 2.7. Let $\varphi: \mathbb{N} \rightarrow[1, \infty)$ be an arbitrary increasing function such that $\varphi(k)$ $>c_{\varphi} \varphi(k+1)$ for some constant $c_{\varphi}, 0<c_{\varphi}<1$.

We denote $\mathscr{L}_{\varphi}: \mathfrak{X} \rightarrow[1, \infty)$ the function given by

$$
\mathscr{L}_{\varphi}(x)=\sup _{k \in \mathbb{N}} \frac{1}{\varphi(k)} \sup _{i \in \Lambda_{k}} \mathscr{L}^{(i)}(x)
$$

where

$$
\mathscr{L}^{(i)}(x)=p_{i}^{2} / 2+k q_{i}^{2}+\lambda q_{i}^{4}+1 .
$$

We put $\mathfrak{X}_{\varphi}=\left\{x \in \mathfrak{X} \mid \mathscr{L}_{\varphi}(x)<+\infty\right\}$.

All the dynamical properties we need in the sequel can be summarized in the following

Theorem 1. i) For all $x \in \mathfrak{X}_{\varphi}$ and $t \in \mathbb{R}^{1}$ the limit

$$
S_{t} x=\lim _{n \rightarrow \infty} S_{t}^{(n)} x
$$

exists in the product topology on $\mathfrak{X} . S_{t} x$ is one parameter group of transformations on $\mathfrak{X}_{\varphi}$ and moreover

$$
\left[\mathscr{L}_{\varphi}\left(S_{t} x\right) \vee \sup _{n} \mathscr{L}_{\varphi}\left(S_{t}^{(n)} x\right)\right] \leqq e^{a|t|} \mathscr{L}_{\varphi}(x)
$$

and for all bounded $\Omega \subset \mathbb{Z}^{v}$.

$$
\mathscr{L}_{\varphi}\left[\tilde{S}_{t}(x)_{\Omega} \cup(x)_{\Omega^{c}}\right] \leqq e^{a|t|} \mathscr{L}_{\varphi}(x)
$$

where a does not depend on $x, t$ and $\Omega$.

ii) If $\varphi^{\prime}: \mathbb{N} \rightarrow[1, \infty)$ is an increasing function such that

$$
\varphi^{\prime}(k)>c_{\varphi^{\prime}} \varphi^{\prime}(k+1) \quad \text { for some } \quad c_{\varphi^{\prime}}, \quad 0<c_{\varphi^{\prime}}<1
$$

and

$$
\begin{aligned}
& \varphi^{\prime}(k) / \varphi(k) \underset{k \rightarrow \infty}{\longrightarrow} 0, \text { then for all } x \in \mathfrak{X}_{\varphi^{\prime}} \\
& \lim _{n \rightarrow \infty} \mathscr{L}_{\varphi}\left(S_{t}^{(n)} x\right)=\mathscr{L}_{\varphi}\left(S_{t} x\right)
\end{aligned}
$$

and for every $x>0$ the convergence in (2.13) is uniform for such $x$ that $\mathscr{L}_{\varphi^{\prime}}(x) \leqq x$. 
iii) Given $x \in \mathfrak{X}_{\varphi^{\prime}}$ the following bounds hold. For any $n$ and $i \in \Lambda_{k}$

$$
\begin{gathered}
\left|q_{i}^{(n)}(t, x)-\bar{q}_{i}(t, x)\right| \vee\left|p_{i}^{(n)}(t, x)-\bar{p}_{i}(t, x)\right| \\
\quad \leqq \frac{\left(a_{1} \varphi(n) \mathscr{L}_{\varphi}(x)\right)^{n-k+1}}{(n-k) !} \quad n>k .
\end{gathered}
$$

Here $\left(q_{i}^{(n)}(t, x), p_{i}^{(n)}(t, x)\right)$ denotes the coordinate and the momentum of the $i$-oscillator in $S_{t}^{(n)} x,\left(\bar{q}_{i}(t, x), \bar{p}_{i}(t, x)\right)$ the coordinate and the momentum of the $i$-oscillator either in $S_{t}^{\left(n^{\prime}\right)} x$ or in $\tilde{S}_{t}(x)_{\Lambda_{n^{\prime}}} \cup x_{\Lambda_{n^{\prime}}}$, with $n^{\prime} \geqq n$ and $a_{1}$ is constant for any fixed $t$.

For any $k, s, n \in \mathbb{N}$ with $s<k<n$ and $x_{\Lambda_{s}}, x_{\Lambda_{s}}^{\prime} \in \mathfrak{X}_{\Lambda_{s}}, i \in \Lambda_{n} \backslash \Lambda_{k}$ :

$$
\begin{aligned}
\mid \bar{q}_{i}^{(n)}( & \left.t, x_{\Lambda_{s}} \cup(x)_{\Lambda_{s}}\right)-\bar{q}_{i}^{(n)}\left(t, x_{\Lambda_{s}}^{\prime} \cup(x)_{\Lambda_{s}}\right) \mid \\
& \vee\left|\bar{p}_{i}^{(n)}\left(t, x_{\Lambda_{s}} \cup(x)_{\Lambda_{s}^{c}}\right)-\bar{p}_{i}^{(n)}\left(t, x_{\Lambda_{s}}^{\prime} \cup(x)_{\Lambda_{s}}\right)\right| \\
\leqq & \frac{\left(a_{2}\left[\mathscr{L}_{\varphi}\left(x_{\Lambda_{s}} \cup(x)_{\Lambda_{\xi}}\right) \vee \mathscr{L}_{\varphi}\left(x_{\Lambda_{s}}^{\prime} \cup(x)_{\Lambda_{s}}\right)\right] \varphi(2 k)\right)^{k-s+1}}{(k-s) !} .
\end{aligned}
$$

Here $\bar{q}_{i}^{(n)}(t, x)$ and $\bar{p}_{i}^{(n)}(t, x)$ denote the coordinate and the momentum of the $i$-oscillator either in $S_{t}^{(n)} x$ or in $\bar{S}_{t}(x)_{A_{n}} \cup x_{A_{n}^{c}}$ and $a_{2}$ is constant for any fixed $t$.

For any $k, n, n^{\prime} \in \mathbb{N}$ such that $k<n, n^{\prime}$ denoting $\left(q_{i}^{(m, \Lambda)}(t, x), p_{i}^{(m, \Lambda)}(t, x)\right)$ the coordinate and the momentum of the $i$-oscillator in $\left(\tilde{S}_{t} x_{\Lambda_{m} \backslash \Lambda}\right) \cup\left(x_{\left[\Lambda_{m} \backslash \Lambda\right]^{c}}\right)$, such that $i \in \Lambda_{k} \backslash \Lambda_{k-1}$ and $\Lambda_{k} \supset \Lambda, \Lambda^{\prime}$ then:

$$
\begin{aligned}
& \left|q_{i}^{(n, \Lambda)}(t, x)-q_{i}^{\left(n^{\prime}, \Lambda^{\prime}\right)}(t, x)\right| \vee\left|p_{i}^{(n, \Lambda)}(t, x)-p_{i}^{\left(n^{\prime}, \Lambda^{\prime}\right)}(t, x)\right| \\
& \quad \leqq \frac{\left(a_{3} \varphi\left(n \vee n^{\prime}\right) \mathscr{L}_{\varphi}(x)^{d+1}\right.}{d !}
\end{aligned}
$$

where

$$
d=\left\{\begin{array}{lccc}
\min \left(n-k, n^{\prime}-k\right) & \text { if } & \Lambda^{\prime}=\Lambda & n \neq n^{\prime} \\
\min \left(k-s, k-s^{\prime}\right) & \text { if } & \Lambda^{\prime} \neq \Lambda & n=n^{\prime} \\
\min \left(k-s, k-s^{\prime}, n-k, n^{\prime}-k\right) & \text { if } & \Lambda \neq \Lambda^{\prime} & n \neq n^{\prime} \\
\infty & \text { if } & n=n^{\prime} & \Lambda=\Lambda^{\prime} .
\end{array}\right.
$$

$a_{3}$ is constant for any fixed $t$, and $s, s^{\prime}=\min \left\{l \in \mathbb{N} \mid \Lambda_{l} \supset \Lambda, \Lambda^{\prime}\right\}, s, s^{\prime}<k$.

The ideas of the Theorem 1 are essentially contained in [3]. We outline the proof in the Appendix.

In this paper we study the evolution

$$
\mu_{t}=S_{t}^{*} \mu=\mu\left(S_{-t} \cdot\right)
$$

of a Gibbs $\mu$ w.r.t. a generating function $h$ which satisfies the following conditions:

1) There exists a constant $c>0$ such that for every finite $\Lambda \subset \mathbb{Z}^{v}$ and $x_{\Lambda} \subset \mathfrak{X}_{\Lambda}$

$$
h\left(x_{\Lambda}\right) \geqq c H\left(x_{\Lambda}\right) .
$$


2) There exists an integer $r$ and a function $\psi:[0, r) \rightarrow \mathbb{R}^{+}$such that for any finite $\Lambda, \Lambda^{\prime} \subset \mathbb{Z}^{v}$ with $\Lambda \cap \Lambda^{\prime}=\Phi$ and all $x_{\Lambda} \in \mathfrak{X}_{\Lambda}$ and $x_{\Lambda^{\prime}}^{\prime} \in \mathfrak{X}_{\Lambda^{\prime}}$ one has :

$$
\begin{aligned}
h\left(x_{\Lambda} \mid x_{\Lambda^{\prime}}^{\prime}\right) & =h\left(x_{\Lambda} \mid x_{\partial \Lambda \cap \Lambda^{\prime}}\right) \\
\left|h\left(x_{\Lambda} \mid x_{\Lambda^{\prime}}^{\prime}\right)\right| & \leqq \sum_{i \in \Lambda} \sum_{i^{\prime} \in \Lambda^{\prime}} \psi\left(\left|i-i^{\prime}\right|\right)\left[\mathscr{L}^{(i)}\left(x_{\Lambda}\right)+\mathscr{L}^{\left(i^{\prime}\right)}\left(x_{\Lambda^{\prime}}^{\prime}\right)\right]
\end{aligned}
$$

where $\partial \Lambda=\left\{i \in \mathbb{Z}^{\nu} \backslash \Lambda|\exists j \in \Lambda:| i-j \mid \leqq r\right\}$.

Here $\mathscr{L}^{(i)}\left(x_{\Omega}\right)$ is defined via $(2.10)$ replacing $x$ by $x_{\Omega}$.

3) Finally, we require that there exists a constant $\bar{c}$ such that:

$$
\begin{gathered}
\left|h\left(x_{\Lambda}\right)-h\left(x_{\Lambda}^{\prime}\right)\right| \leqq \bar{c}|\Lambda| \max _{i \in \Lambda}\left[\mathscr{L}^{(i)}\left(x_{\Lambda}\right) \vee \mathscr{L}^{(i)}\left(x_{\Lambda}^{\prime}\right)\right] \\
\cdot \max _{i \in \Lambda}\left[\left|q_{i}-q_{i}^{\prime}\right| \vee\left|p_{i}-p_{i}^{\prime}\right|\right] .
\end{gathered}
$$

In the Appendix we prove:

Theorem 2. Let h satisfy the condition 1)-3) above. Then there exists at least one Gibbs state $\mu$ corresponding to $h$ such that

$$
\mu\left(\mathfrak{X}_{\varphi^{\prime}}\right)=1
$$

for $\varphi^{\prime}(k)=(\log k) \vee 1$.

Theorem 2 allows us to define the time evolved state $\mu_{t}$ via equality (2.18).

Now we can formulate the main result of this paper.

Theorem 3. Let $\mu$ be a Gibbs state corresponding to a generating function $h$ satisfying the condition 1)-3), and such that (2.22) holds. Then $\mu_{t}$ is a Gibbs state corresponding to the generating function $h_{t}$ given by

$$
h_{t}\left(x_{\Lambda}\right)=h\left(\tilde{S}_{-t} x_{A}\right)
$$

where $\Lambda \subset \mathbb{Z}^{v}$ is finite.

Remark. A natural question arising in the examination of Theorem 3 is if the condition 1)-3) on the initial $\mu$ are preserved during the motion. Condition 1 which means superstability for $h$ is obviously preserved by the conservation of energy, with the same coefficient $c$. A sort of local Lipschitz condition as (2.21) can also exhibited for $h_{t}$, by the use of Theorem 1.

Furthermore one can prove, by the use of the same ideas of Lemma 3.2 below, that condition $\left(2.20^{\prime}\right)$ is preserved for $h_{t}$ with a function $\psi_{t}$ (of course no more with compact support because dynamics destroys locality) more than exponentially decreasing at infinity. This will imply that the superstable estimates (Ref. [3] and A.II below) hold for $\mu_{t}$. Obviously condition (2.20) is no more preserved.

\section{Proofs}

In order to prove Theorem 3 we have to give good estimates on the quantities $h_{t}\left(x_{A} \mid(x)_{\Lambda^{c}}\right)$ and $\Xi_{\Lambda}\left(h_{t} ; x\right)$ for a sufficiently large set of $x \in \mathfrak{X}$. While the first quantity may be estimated by the use of Theorem 1, it seems hard to have a good control of $\Xi_{\Lambda}\left(h_{t} ; x\right)$ in terms of $x$ by brute force using the dynamical properties we know. We 
do not approach the problem directly, but we shall prove an analog of Theorem 3 (see Theorem 4 below) where the conditional probabilities are taken not w.r.t. $\xi_{A}$ but w.r.t. its refinement $\xi_{A}^{m}$ whose atoms may be identificated with proper subsets of $\mathfrak{X}_{A}$ of finite Lebesgue measure, which will allow us to avoid problems on the convergence of the normalization factor. Once Theorem 4 is proved, it is not hard to prove Theorem 3. We shall start by giving the new partition and a precise formulation of Theorem 4 . Then we shall show the passage from Theorem 4 to Theorem 3. The rest of this section will be devoted to the proof of Theorem 4 .

For all $m \in \mathbb{N}$ we define the partition $\xi_{A}^{m}$ of $\mathfrak{X}_{\varphi}$ by giving its atoms:

$$
\begin{aligned}
a_{\Lambda}^{m}(x)= & \left\{y \mid(y)_{\Lambda^{c}}=(x)_{\Lambda^{c}} ;\right. \\
& \left.\mathscr{L}_{\varphi}(y)<m \quad \text { if } \quad \mathscr{L}_{\varphi}(x)<m \quad\left[\mathscr{L}_{\varphi}(y)\right]=\left[\mathscr{L}_{\varphi}(y)\right] \quad \text { otherwise }\right\}
\end{aligned}
$$

where

$$
\varphi(k)=1 \vee \log ^{2} k \quad k \in \mathbb{N}
$$

where $[x]$ denotes the integer part of $x \in \mathbb{R} . \xi_{A}^{m}$ is obviously measurable. Each atom $a_{\Lambda}^{m}(x)$ induces the subset $\tilde{a}_{A}^{m}(x) \subset \mathfrak{X}_{\Lambda}$ :

$$
\begin{aligned}
\tilde{a}^{m}(x)= & \left\{y_{A} \mid \mathscr{L}_{\varphi}\left(y_{\Lambda} \cup(x)_{\Lambda^{c}}\right)<m \text { if } \mathscr{L}_{\varphi}(x)<m\right. \\
& {\left.\left[\mathscr{L}_{\varphi}\left(\left(y_{A}\right) \cup(x)_{A^{c}}\right)\right]=\left[\mathscr{L}_{\varphi}(x)\right] \quad \text { otherwise }\right\} }
\end{aligned}
$$

and for each state $\mu$, the family $\left\{\mu\left(\cdot \mid a_{A}^{m}(x)\right)\right\}$ induces the family of measures (still denoted by $\mu\left(\cdot \mid a_{\Lambda}^{m}(x)\right)$ supported in corresponding $\left.\tilde{a}_{\Lambda}^{m}(x)\right)$.

The main point of the proof of Theorem 3 in the following:

Theorem 4. Let $h$ be a function satisfying the condition 1)-3) and $\mu$ be a Gibbs state corresponding to $h$ and satisfying (2.20). Then for any $m \in \mathbb{N}$ we have:

$$
\mu_{t}\left(A \mid a_{A}^{m}(x)\right)=\frac{\int_{A} d \lambda(\tilde{x}) \exp \left[-h_{t}\left(\tilde{x}_{A}\right)-h_{t}\left(\tilde{x}_{A} \mid(x)_{A^{c}}\right)\right]}{\left.\int_{a_{A}^{m}(x)} d \lambda(\tilde{x}) \exp \left[-h_{t}\left(\tilde{x}_{A}\right)-h_{t}\left(\tilde{x}_{A}\right)(x)_{\Lambda^{c}}\right)\right]}
$$

where $A \subset \tilde{a}_{\Lambda}^{m}(x)$ is a Borel set and $h_{t}$ is defined by $(2.21)$.

Proof of Theorem 3. Let us consider two bounded measurable sets $A$ and $B$ in $\mathfrak{X}_{A}$ such that $\lambda(A), \lambda(B)$ are different from zero, and a sufficiently large $m$ such that $A, B \subset \tilde{a}_{A}^{m}(x)$ for all $x \in \mathfrak{X}_{\varphi}$ with $\mathscr{L}_{\varphi}(x)<m$. Since $\xi_{A}^{m}$ is a refinement of $\xi_{A}$, it results by (2.2):

$$
\mu_{t}\left(A \mid a_{\Lambda}^{m}(x)\right) \mu_{t}\left(\tilde{a}_{\Lambda}^{m}(x) \mid(x)_{A^{c}}\right)=\mu_{t}\left(A \mid(x)_{A^{c}}\right)
$$

and an analogous expression for $B$ which hold for $\mu_{t}$-a.a. $x$ such that $\mathscr{L}_{\varphi}(x)<m$. By (3.3), both $\mu_{t}\left(A \mid a_{\Lambda}^{m}(x)\right)$ and $\mu_{t}\left(B \mid a_{\Lambda}^{m}(x)\right)$ are different from zero and:

$$
\frac{\mu_{t}\left(A \mid(x)_{\Lambda^{c}}\right)}{\mu_{t}\left(A \mid a_{\Lambda}^{m}(x)\right)}=\frac{\mu_{t}\left(B \mid(x)_{\Lambda^{c}}\right)}{\mu_{t}\left(B \mid a_{\Lambda}^{m}(x)\right)} .
$$

By the use of (3.3), we obtain:

$$
\begin{aligned}
& \mu_{t}\left(A \mid(x)_{A^{c}}\right) \int_{B} e^{-\left[h_{t}\left(\tilde{x}_{A}\right)+h_{t}\left(\tilde{x}_{A} \mid(x)_{\Lambda^{c}}\right)\right]} d \lambda\left(\tilde{x}_{A}\right) \\
& \quad=\mu_{t}\left(B \mid(x)_{\Lambda^{c}}\right) \int_{A} e^{-\left[h_{t}\left(\tilde{x}_{A}\right)+h_{t}\left(\tilde{x}_{A} \mid(x)_{\left.A^{c}\right)}\right)\right.} d \lambda\left(\tilde{x}_{A}\right) \\
& \text { for } \mu_{t} \text { - } \text { a.a. } x \in \mathfrak{X}_{\varphi^{\prime}}
\end{aligned}
$$


Consider now an increasing countable family of $B$ 's invading $\mathfrak{X}_{A}$. There exists a $\mu_{t}$ full measure set $\tilde{\mathfrak{X}}_{\varphi}$ such that (3.6) holds simultaneously for all elements of the family. Fixed $x \in \tilde{\mathfrak{X}}_{\varphi}$, it is possible to find a sufficiently large $B$ for which the r.h.s. of (3.6) is different from 0 . This implies that $\mu_{t}\left(A \mid(x)_{A^{c}}\right) \neq 0$. Finally, by taking the limit $B \rightarrow \mathfrak{X}_{A}$ we obtain simultaneously:

$$
\begin{aligned}
\mu_{t}\left(A \mid(x)_{A^{c}}\right) & =\frac{\int_{A} d \lambda\left(\tilde{x}_{A}\right) \exp \left[-h_{t}\left(\tilde{x}_{A}\right)-h_{t}\left(\tilde{x}_{A} \mid(x)_{A^{c}}\right)\right]}{\Xi_{A}\left(h_{t} ; x\right)} \\
\Xi_{\Lambda}\left(h_{t} ; x\right) & =\int_{\tilde{x}_{A}} d \lambda\left(\tilde{x}_{A}\right) \exp \left[-h_{t}\left(\tilde{x}_{A}\right)-h_{t}\left(\tilde{x}_{A} \mid(x)_{A^{c}}\right)\right]<+\infty
\end{aligned}
$$

that proves Theorem 3.

Proof of Theorem 4. We shall consider the following measure:

$$
\mu_{t}^{n}=S_{t}^{(n) *} \mu=\mu\left(S_{-t}^{n} \cdot\right)
$$

where $\mu$ from now on is the same measure as in Theorem 3 .

We denote by $\mu_{t}^{n}\left(\cdot\left\{a_{\Lambda}^{m}(x)\right)\right.$ its conditional probability w.r.t. $\xi_{\Lambda}^{n}$. Then:

Lemma 3.1. For every $x \in \mathfrak{X}_{\varphi}$ and $n$ such that $\Lambda_{n} \supset \Lambda$, there exists the Radon-Nicodym derivative:

$$
P_{t}^{n}\left(x_{\Lambda} \mid a_{\Lambda}^{m}(x)\right)=\frac{d \mu_{t}^{n}\left(x_{\Lambda} \mid a_{\Lambda}^{m}(x)\right)}{d \lambda\left(x_{\Lambda}\right)}
$$

which is given by:

$$
P_{t}^{n}\left(x_{A} \mid a_{\Lambda}^{m}(x)\right)=\frac{\exp \left[-h\left(\left(S_{-t}^{(n)}\left(x_{A} \cup(x)_{\Lambda^{c}}\right)_{\Lambda_{n+r}}\right)\right]\right.}{\int_{a_{A}^{m}(x)} \exp \left[-h\left(\left(S_{-t}^{(n)}\left(x_{\Lambda} \cup(x)_{A^{c}}\right)_{A_{n+r}}\right)\right]\right.}
$$

where $r$ is the same as in condition 2).

Proof. The estimate (2.19) and the conservation of energy

$$
H\left[\left(S_{-t}^{(n)}\left(x_{A} \cup(x)_{\Lambda^{c}}\right)\right)_{\Lambda_{n+r}}\right]=H\left[\left(x_{\Lambda} \cup(x)_{\Lambda^{c}}\right)_{\Lambda_{n+r}}\right]
$$

imply that the integral in r.h.s. of (3.10) is uniformly bounded on $m$. Furthermore, by condition 2) one can prove that:

$$
\begin{gathered}
P_{t}^{n}\left(x_{\Lambda_{n}} \mid(x)_{\Lambda_{n}^{c}}\right)=\frac{e^{\left.-h\left(\left(S_{-t}^{(n)}\left(x_{\Lambda_{n}} \cup(x)\right)_{\Lambda_{n}^{c}}\right)\right)_{\Lambda_{n}}\right)-h\left(\left(S_{-t}^{(n)}\left(x_{\Lambda_{n}} \cup(x)_{\left.\Lambda_{n}^{c}\right)}\right) \Lambda_{\Lambda_{n}} \mid x_{\Lambda_{n}^{c}}\right)\right.}}{\text { normalization factor }} \\
=\frac{\exp \left[-h\left(\left(S_{-t}^{(n)}\left(x_{\Lambda_{n}} \cup(x)_{\Lambda_{n}^{c}}\right)_{\Lambda_{n+r}}\right)\right]\right.}{\int_{\mathfrak{X}_{\Lambda_{n}}} d \lambda\left(\tilde{x}_{\Lambda_{n}}\right) \exp \left[-h\left(\left(S_{-t}^{(n)}\left(x_{\Lambda_{n}} \cup(x)_{\Lambda_{n}^{c}}\right)_{\Lambda_{n+r}}\right)\right]\right.}
\end{gathered}
$$

where $P_{t}^{n}\left(x_{\Lambda_{n}} \mid(x)_{\Lambda_{n}^{c}}\right)$ is the Radon-Nikodym derivative $\frac{d \mu_{t}^{n}\left(x_{\Lambda} \mid(x)_{\Lambda_{n}^{c}}\right)}{d \lambda\left(x_{\Lambda}\right)}$.

Since $\xi_{A}$ is a refinement of $\xi_{A_{n}}$, we apply (2.2) and deduce that the density $P_{t}^{n}\left(x_{\Lambda} \mid(x)_{\Lambda^{c}}\right)$ has the same form as $P_{t}^{n}\left(x_{\Lambda_{n}} \mid(x)_{\Lambda_{n}^{c}}\right)$ with the normalization factor, obtained by integrating the coordinates in $\mathfrak{X}_{\Lambda}$. Still using (2.2), we obtain that the 
density $P_{t}^{n}\left(x_{\Lambda} \mid a_{A}^{m}(x)\right)$ and momenta has the same form as $P_{t}^{n}\left(x_{\Lambda} \mid(x)_{\Lambda^{c}}\right)$ with the normalization factor obtained by integrating on $\tilde{a}_{A}^{m}(x)$. So (3.10) is proved.

Lemma 3.2. For any $x \in \mathfrak{X}_{\varphi}$ and $x_{\Lambda} \in \tilde{a}_{\Lambda}^{m}(x)$ there exists the limit:

$$
P_{t}^{(\infty)}\left(x_{\Lambda} \mid a_{\Lambda}^{m}(x)\right)=\lim _{n \rightarrow \infty} P_{t}^{n}\left(x_{\Lambda} \mid a_{\Lambda}^{m}(x)\right) .
$$

Moreover there exists functions $\gamma_{m}, \tilde{\gamma}_{m}: \mathbb{N} \times \mathfrak{X} \rightarrow \mathbb{R}$ with

$$
\lim _{n \rightarrow \infty} \gamma_{m}(n ; x)=\lim _{n \rightarrow \infty} \tilde{\gamma}_{m}(n ; x)=0
$$

such that for all $n^{\prime} \geqq n$

$$
\exp \left[-\gamma_{m}(n ; x)\right] \leqq \frac{P_{t}^{n}\left(x_{\Lambda} \mid a_{\Lambda}^{m}(x)\right)}{P_{t}^{n^{\prime}}\left(x_{\Lambda} \mid a_{\Lambda}^{m}(x)\right)} \leqq \exp \left[\gamma_{m}(n ; x)\right]
$$

and $\gamma_{m}\left(n ; S_{t}^{\left(n^{\prime}\right)} x\right) \leqq \tilde{\gamma}_{m}(n, x)$.

Proof. We check that

$$
\lim _{\substack{n \rightarrow \infty \\ n^{\prime}>n}} \frac{P_{t}^{n}\left(x_{\Lambda} \mid a_{\Lambda}^{m}(x)\right)}{P_{t}^{n^{\prime}}\left(x_{\Lambda} \mid a_{\Lambda}^{m}(x)\right)}=1
$$

Suppose $n^{\prime}>n, \tilde{x}_{\Lambda}, \tilde{x}_{\Lambda}^{\prime} \in \mathfrak{X}_{\Lambda}$ are fixed, and consider the ratio:

$$
\frac{\exp \left[-h\left(\left(S_{-t}^{(n)}\left(\tilde{x}_{\Lambda} \cup(x)_{\Lambda^{c}}\right)\right)_{\Lambda_{n+r}}\right)\right] \exp \left[-h\left(\left(S_{-t}^{\left(n^{\prime}\right)}\left(\tilde{x}_{\Lambda}^{\prime} \cup(x)_{A^{c}}\right)\right)_{A_{n^{\prime}+r}}\right)\right]}{\exp \left[-h\left(\left(S_{-t}^{(n)}\left(\tilde{x}_{\Lambda}^{\prime} \cup(x)_{A^{c}}\right)\right)_{A_{n+r}}\right)\right] \exp \left[-h\left(\left(S_{-t}^{\left(n^{\prime}\right)}\left(\tilde{x}_{A} \cup(x)_{A^{c}}\right)\right)_{\Lambda_{n^{\prime}+r}}\right)\right]} .
$$

It is convenient to consider a sublattice of $\mathbb{Z}^{v}$ constructed by cells of side $r$. We denote these cells $I_{i}, i \in \mathbb{Z}^{v}$. We put:

$$
\tilde{Y}_{h, i}=\left(S_{-t}^{(h)}\left(\tilde{x}_{A} \cup(x)_{A^{c}}\right)\right)_{I_{\imath} \cap A_{h+r}} ; \quad \tilde{Y}_{n, i}^{\prime}=\left(S_{-t}^{(h)}\left(\tilde{x}_{\Lambda}^{\prime} \cup(x)_{A^{c}}\right)\right)_{I_{i} \cap A_{h+r}}
$$

where $h=n, n^{\prime}$.

In virtue of Condition 2), (3.17) become:

$$
\frac{\exp -\left[\sum_{i} h\left(\tilde{Y}_{n, i}\right)+\sum_{i_{1}, i_{2}}^{\prime} h\left(\tilde{Y}_{n, i_{1}} \mid \tilde{Y}_{n, i_{2}}\right)\right] \exp -\left[\sum_{i} h\left(\tilde{Y}_{n^{\prime}, i}^{\prime}\right)+\sum_{i_{1}, i_{2}}^{\prime} h\left(\tilde{Y}_{n^{\prime}, i_{1}}^{\prime} \mid \tilde{Y}_{n^{\prime}, i_{2}}^{\prime}\right)\right]}{\exp -\left[\sum_{i} h\left(\tilde{Y}_{n, i}^{\prime}\right)+\sum_{i_{1}, i_{2}}^{\prime} h\left(\tilde{Y}_{n, i_{1}}^{\prime} \mid \tilde{Y}_{n, i_{2}}^{\prime}\right)\right] \exp -\left[\sum_{i} h\left(Y_{n, i}\right)+\sum_{i_{1}, i_{2}}^{\prime} h\left(\tilde{Y}_{n^{\prime}, i_{1}} \mid \tilde{Y}_{n^{\prime}, i_{2}}\right)\right]}
$$

where $\sum$ ' means the sum on the nearest neighbours.

Now we compare:

a) $h\left(\tilde{Y}_{n, i}\right)$ with $h\left(\tilde{Y}_{n, i}^{\prime}\right)$ and $h\left(\tilde{Y}_{n^{\prime}, i}\right)$ with $h\left(\tilde{Y}_{n^{\prime}, i}^{\prime}\right)$ for $I_{i} \notin \Lambda_{n / 2}$

b) $h\left(\tilde{Y}_{n, i}\right)$ with $h\left(\tilde{Y}_{n, i}\right)$ and $h\left(\tilde{Y}_{n, i}^{\prime}\right)$ with $h\left(\tilde{Y}_{n^{\prime}, i}^{\prime}\right)$ for $I_{i} \subset \Lambda_{n / 2}$. 
Then, by condition 3) (see (2.19)) and Theorem 1, iii) (see (2.15)), we have for $I_{i} \notin A_{n / 2}$ :

$$
\begin{aligned}
& \left|h\left(\tilde{Y}_{n, i}\right)-h\left(\tilde{Y}_{n, \varepsilon}^{\prime}\right)\right| v\left|h\left(\tilde{Y}_{n^{\prime}, i}\right)-h\left(\tilde{Y}_{n^{\prime}, i}^{\prime}\right)\right| \\
& \quad \leqq c^{\prime} \max _{j \in I_{1}}\left[\mathscr{L}^{(j)}\left(\tilde{Y}_{n, i}\right) v \mathscr{L}^{(j)}\left(\tilde{Y}_{n, i}^{\prime}\right) v \mathscr{L}^{(j)}\left(\tilde{Y}_{n^{\prime}, i}\right) v \mathscr{L}^{(j)}\left(\tilde{Y}_{n^{\prime}, i}^{\prime}\right)\right] \\
& \quad \cdot \frac{\left[a_{2}\left(\mathscr{L}_{\varphi}\left(\tilde{x}_{\Lambda} \cup(x)_{\Lambda^{c}}\right) v \mathscr{L}_{\varphi}\left(\tilde{x}_{\Lambda}^{\prime} \cup(x)_{\Lambda^{c}}\right)\right) \varphi(n+2 r)\right]^{\frac{n}{2}+d(\Lambda)-r+1}}{\left(\frac{n}{2}-d(\Lambda)-r\right) !}
\end{aligned}
$$

where $c^{\prime}>0$ is a constant and $d(\Lambda)=\min \left\{k \mid \Lambda_{k} \supset \Lambda\right\}$.

Analogously, by Theorem 1 iii) (see (2.14), we have for $I_{i} \subset A_{n / 2}$ :

$$
\begin{aligned}
& \left|h\left(\tilde{Y}_{n, i}\right)-h\left(\tilde{Y}_{n^{\prime}, i}\right)\right| v\left|h\left(\tilde{Y}_{n, i}^{\prime}\right)-h\left(\tilde{Y}_{n^{\prime}, i}^{\prime}\right)\right| \\
& \leqq c^{\prime} \max _{j \in I_{2}}\left[\mathscr{L}^{(j)}\left(\tilde{Y}_{n, i}\right) v \mathscr{L}^{(j)}\left(\tilde{Y}_{n, i}^{\prime}\right) v \mathscr{L}^{(j)}\left(\tilde{Y}_{n^{\prime}, i}\right) v \mathscr{L}^{(j)}\left(\tilde{Y}_{n^{\prime}, i}^{\prime}\right)\right] \\
& . \frac{\left[a_{1}\left(\mathscr{L}_{\varphi}\left(\left(\tilde{x}_{\Lambda} \cup(x)_{\Lambda^{c}}\right)\right) v \mathscr{L}_{\varphi}\left(\left(\tilde{x}_{\Lambda}^{\prime} \cup(x)_{\Lambda^{c}}\right)\right)\right) \cdot \varphi(n)\right]^{n / 2+1}}{\left(\frac{n}{2}\right) !} .
\end{aligned}
$$

Furthermore, Theorem 1, i) (see (2.12)) gives:

$$
\mathscr{L}^{(j)}\left(\tilde{Y}_{n, i}\right) \leqq \varphi(j) \mathscr{L}_{\varphi}\left(S_{-t}^{(n)}\left(\tilde{x}_{\Lambda} \cup(x)_{\Lambda^{c}}\right)\right) \leqq e^{a|t|} \varphi(j) \mathscr{L}_{\varphi}\left(\tilde{x}_{\Lambda} \cup(x)_{\Lambda^{c}}\right)
$$

and similar bounds on the other $\mathscr{L}_{\varphi}^{(j)}$ 's comparing in (3.20) and (3.21).

Hence, there exists a function $\gamma_{1}\left(n, \tilde{x}_{A}, \tilde{x}_{\Lambda}^{\prime}, x\right)$ such that:

$$
\left|\sum_{i} h\left(\tilde{Y}_{n, i}\right)-\sum_{i} h\left(\tilde{Y}_{n, i}^{\prime}\right)+\sum_{i} h\left(\tilde{Y}_{n^{\prime}, i}^{\prime}\right)-\sum_{i} h\left(\tilde{Y}_{n^{\prime}, i}\right)\right| \leqq \gamma,\left(n, \tilde{x}_{\Lambda}, \tilde{x}_{\Lambda}^{\prime}, x\right)
$$

and, because of the definition of $\varphi\left(\right.$ see $\left.\left(3.1^{\prime}\right)\right)$,

$$
\begin{aligned}
\gamma_{1}(n, x) \equiv & \sup \left\{\gamma_{1}\left(n, \tilde{x}_{\Lambda}, \tilde{x}_{\Lambda}^{\prime}, x\right) \mid x \in \mathfrak{X}_{\varphi}, \tilde{x}_{\Lambda}, \tilde{x}_{\Lambda}^{\prime} \in \mathfrak{X}_{\Lambda},\right. \\
& \left.\cdot \mathscr{L}_{\varphi}\left(\tilde{x}_{\Lambda} \cup(x)_{\Lambda^{c}}\right) \cup \mathscr{L}_{\varphi}\left(\tilde{x}_{\Lambda}^{\prime} \cup(x)_{\Lambda^{c}}\right) \leqq x\right\}
\end{aligned}
$$

verifies :

$$
\lim _{n \rightarrow \infty} \gamma_{1}(n, x)=0 \text {. }
$$

A similar estimate may be obtained for:

$$
\begin{aligned}
& \left.\| \sum_{i_{1}, i_{2}}^{\prime} h\left(\tilde{Y}_{n, i_{1}} \mid \tilde{Y}_{n, i_{2}}\right)-\sum_{i_{1}, i_{2}}^{\prime} h\left(\tilde{Y}_{n, i_{1}}^{\prime} \mid \tilde{Y}_{n, i_{2}}^{\prime}\right)\right] \\
& \quad+\left[\sum_{i_{2}, i_{2}}^{\prime} h\left(\tilde{Y}_{n^{\prime}, i_{1}}^{i} \mid \tilde{Y}_{n^{\prime}, i_{2}}^{\prime}\right)-\sum_{i_{1}, i_{2}}^{\prime} h\left(\tilde{Y}_{n^{\prime}, i_{1}} \mid \tilde{Y}_{n^{\prime}, i_{2}}\right)\right] .
\end{aligned}
$$

Hence, ratio (3.17) is bounded from above by $e^{2 \gamma_{1}(n, x)}$ and from below by $e^{-2 \gamma_{1}(n, x)}$ where $\tilde{x}_{A}, \tilde{x}_{A}^{\prime}$ and $x$ are chosen so that:

$$
\mathscr{L}_{\varphi}\left(\tilde{x}_{A} \cup(x)_{\Lambda^{c}}\right) v \mathscr{L}_{\varphi}\left(\tilde{x}_{\Lambda}^{\prime} \cup(x)_{\Lambda^{c}}\right) \leqq x .
$$


Now if $\tilde{x}_{A}, \tilde{x}_{A}^{\prime} \in \tilde{a}_{A}^{(m)}(x)$, then there exists a function $\gamma_{m}(n ; x)$ satisfying (3.14) and such that $e^{ \pm \gamma_{m}(n ; x)}$ bounds the ratio (3.17).

Integrating these estimate on $d \lambda\left(\tilde{x}_{\Lambda}^{\prime}\right)$ over $\tilde{a}_{\Lambda}^{m}(x)$, one finally gets:

$$
\exp \left[-\gamma_{m}(n ; x)\right] \leqq \frac{P_{t}^{(n)}\left(\tilde{x}_{\Lambda} \mid a_{\Lambda}^{m}(x)\right)}{P_{t}^{\left(n^{\prime}\right)}\left(\tilde{x}_{\Lambda} \mid a_{\Lambda}^{m}(x)\right)} \leqq \exp \left[\gamma_{m}(n ; x)\right]
$$

and hence (3.13) and (3.15). The existence of $\tilde{\gamma}_{m}(n ; x)$ easily follows by Theorem $\left.1, i\right)$.

Lemma 3.3. The function $P_{t}^{(\infty)}\left(\cdot \mid a_{A}^{m}(x)\right)$ defined by (3.13) satisfies the equality:

$$
P_{t}^{(\infty)}\left(x_{\Lambda} \mid a_{\Lambda}^{m}(x)\right)=\frac{\exp \left[-h_{t}\left(x_{1}\right)-h_{t}\left(x_{\Lambda} \mid(x)_{\Lambda^{c}}\right)\right]}{\int_{\tilde{a}_{\Lambda}^{m}(x)} d \lambda\left(x_{\Lambda}^{\prime}\right) \exp \left[-h_{t}\left(x_{\Lambda}^{\prime}\right)-h_{t}\left(x_{\Lambda}^{\prime} \mid(x)_{\Lambda^{c}}\right)\right]} .
$$

In particular the r.h.s. of (3.28) makes sense.

Proof. First of all we show the existence of $h_{t}\left(x_{1} \mid(x)_{\Lambda^{c}}\right)$. To this purpose it is enough to prove:

$$
\lim _{\substack{n \rightarrow \infty \\ n^{\prime}>n}}\left\{h_{t}\left(x_{\Lambda_{n}}\right)-h_{t}\left(x_{A_{n^{\prime}}}\right)-h_{t}\left(x_{A_{n} \backslash \Lambda}\right)+h_{t}\left(x_{\Lambda_{n^{\prime} \backslash \Lambda}}\right)\right\}=0 .
$$

We only sketch the proof of (3.29) since it uses the same ideas as the proof of Lemma 3.1. We define $Z_{n, i}=\left(\tilde{S}_{-t} x_{A_{n}}\right)_{I_{2} \cap A_{n}}, \bar{Z}_{n, i}=\left(\tilde{S}_{-t} x_{\Lambda_{n} \backslash A}\right)_{I_{\imath} \cap A_{n} \backslash \Lambda}, Z_{n^{\prime}, i}$ and $\bar{Z}_{n^{\prime}, i}$ are defined analogously. Then:

$$
\begin{aligned}
& \left|h_{t}\left(x_{\Lambda_{n}}\right)-h_{t}\left(x_{\Lambda_{n^{\prime}}}\right)-h_{t}\left(x_{\Lambda_{n} \backslash A}\right)+h_{t}\left(x_{\Lambda_{n^{\prime} \backslash \Lambda}}\right)\right| \\
& \leqq\left|\sum_{i} h\left(Z_{n, i}\right)-\sum_{i} h\left(Z_{n^{\prime}, i}\right)-\sum_{i} h\left(\bar{Z}_{n, i}\right)+\sum_{i} h\left(\bar{Z}_{n^{\prime}, i}\right)\right| \\
& +\mid \sum_{i_{1}, i_{2}}^{\prime} h\left(Z_{n, i_{1}} \mid Z_{n, i_{2}}\right)-\sum_{i_{1}, i_{2}}^{\prime} h\left(Z_{h^{\prime}, i_{1}} \mid Z_{n^{\prime}, i_{2}}\right) \\
& \sum_{i_{1}, i_{2}}^{\prime} h\left(\bar{Z}_{n, i_{1}} \mid \bar{Z}_{n, i_{2}}\right)+\sum_{i_{1}, i_{2}}^{\prime} h\left(\bar{Z}_{h^{\prime}, i_{1}} \mid \bar{Z}_{n^{\prime}, i_{2}}\right) \mid \text {. }
\end{aligned}
$$

We compare
a) $h\left(Z_{n, i}\right)$ with $h\left(Z_{n^{\prime}, i}\right)$ if $I_{i} \subset A_{n / 2}$
a) $h\left(\bar{Z}_{n, i}\right)$ with $h\left(\bar{Z}_{n^{\prime}, i}\right)$
b) $h\left(Z_{n, i}\right)$ with $h\left(\bar{Z}_{n, i}\right)$ if $I_{i} \not \subset \Lambda_{n}$
b) $h\left(Z_{n^{\prime}, i}\right)$ with $h\left(\bar{Z}_{n^{\prime}, i}\right)$.

Analog comparison are made for the terms $h(\cdot \mid \cdot)$ 's.

By the use of the estimates (2.16) of Theorem 1, (2.21) of Condition 3, and (2.12) the statement (3.29) can be proved.

The second step is to prove the equality

$$
\begin{aligned}
& \lim _{n \rightarrow \infty} \frac{\exp \left[-h\left(S_{-t}^{(n)}\left(\tilde{x}_{\Lambda} \cup(x)_{\Lambda^{c}}\right)_{\Lambda_{n+r}}\right]\right.}{\exp \left[-h\left(\tilde{S}_{-t}\left(\tilde{x}_{\Lambda} \cup(x)_{\Lambda_{n+r} \backslash \Lambda}\right)\right)\right]} \\
& \frac{\int_{\tilde{a}_{\Lambda}^{m}(x)} d \lambda\left(\tilde{x}_{\Lambda}^{\prime}\right) \exp \left[-h\left(\tilde{S}_{-t}\left(\tilde{x}_{\Lambda}^{\prime} 0(x)_{A_{n+r} \backslash A}\right)\right)\right]}{\int_{\hat{a}_{\Lambda}^{m}(x)} d \lambda\left(\tilde{x}_{\Lambda}\right) \exp \left[-h\left(\left(S_{-t}^{(n)}\left(\tilde{x}_{\Lambda} \cup(x)_{\Lambda^{c}}\right)\right)_{\Lambda_{n+r}}\right)\right]}=1 .
\end{aligned}
$$


As in Lemma 3.2, we consider the ratio

$$
\frac{\exp \left[-h\left(\left(S_{-t}^{(n)}\left(\tilde{x}_{\Lambda} \cup(x)_{\Lambda^{c}}\right)\right)_{\Lambda_{n+r}+r}\right)\right] \exp \left[-h\left(\tilde{S}_{-t}\left(\tilde{x}_{\Lambda}^{\prime} \cup(x)_{\Lambda_{n+r} \backslash \Lambda}\right)\right)\right]}{\exp \left[-h\left(\left(S_{-t}^{(n)}\left(\tilde{x}_{\Lambda}^{\prime} \cup(x)_{\Lambda^{c}}\right)\right)_{\Lambda_{n+r}}\right)\right] \exp \left[-h\left(\tilde{S}_{-t}\left(\tilde{x}_{\Lambda} \cup(x)_{\Lambda_{n+r} \backslash \Lambda}\right)\right)\right]}
$$

and the repeating the construction utilized in Lemma 3.2 we obtain for (3.21) the same bound from above and below by $\exp \left[ \pm \bar{\gamma}_{m}(n ; x)\right]$ where $\bar{\gamma}_{m}$ has the same properties as $\gamma_{m}$. This gives (3.30).

Finally, we observe that in the ratio (3.30) the numerator of the first term being divided by, the denominator of the second gives in the limit $P^{\infty}\left(\cdot \mid a_{\Lambda}^{m}(x)\right)$ by Lemma 3.2 and the remaining term may be written as:

$$
\left(\frac{\exp -\left[h_{t}\left(\tilde{x}_{\Lambda}\right)+h_{t}\left(\tilde{x}_{\Lambda} \mid(x)_{\Lambda_{n+r \backslash \Lambda}}\right)\right]}{\text { normalization factor }}\right)^{-1} \text {. }
$$

Hence, by the existence of $\left.h_{t}\left(x_{A}\right)(x)_{A^{c}}\right)$, the Lemma 3.3 is proved.

Now we are able to prove Theorem 4 by showing the following equality:

$$
\int_{\mathfrak{X}} \mu_{t}(d x) F(x)=\int_{\mathfrak{X}} \mu_{t}(d x) \int_{\tilde{a}_{\Lambda}^{m}(x)} d \lambda\left(x_{\Lambda}\right) P_{t}^{\infty}\left(x_{\Lambda} \mid a_{\Lambda}^{m}(x)\right) F\left(x_{\Lambda} \cup(x)_{\Lambda^{c}}\right) .
$$

It suffices to consider the case where $F$ is cylindrical (i.e. $F$ depends explicitely only on the coordinates and momenta in some finite $\Lambda^{\prime}\left(\mathbb{Z}^{v}\right)$, continuous and bounded. The general case may be obtained by standard approximation arguments. For the brevity of notations we take $\Lambda^{\prime}=\Lambda$ in the calculations below; the reader can easily extend them for arbitrary finite $\Lambda^{\prime}$. Since for every bounded continuous cylindrical F

$$
\int_{\mathfrak{X}} \mu_{t}(d x) F(x)=\lim _{n \rightarrow \infty} \int_{\mathfrak{X}} \mu_{t}^{(n)}(d x) F(x)
$$

to prove (3.32) it suffices to prove:

$$
\begin{array}{r}
\lim _{n \rightarrow \infty} \int_{\mathfrak{X}} \mu_{t}^{(n)}(d x) \int_{\tilde{a}_{\Lambda}^{m}(x)} d \lambda\left(x_{\Lambda}\right) P_{t}^{(n)}\left(x_{\Lambda} \mid a_{\Lambda}^{m}(x)\right) \tilde{F}\left(x_{\Lambda}\right) \\
=\int_{\mathfrak{X}} \mu_{t}(d x) \int_{\hat{a}_{\Lambda}^{m}(x)} d \lambda\left(x_{\Lambda}\right) P_{t}^{\infty}\left(x_{\Lambda} \mid a_{\Lambda}^{m}(x)\right) \tilde{F}\left(x_{\Lambda}\right)
\end{array}
$$

where $\tilde{F}: \mathfrak{X}_{A} \rightarrow \mathbb{R}$ is defined by $F(x)=\tilde{F}\left((x)_{A}\right)$.

By the use of an $\varepsilon / 3$ argument we show (3.34) by proving that the following three terms below are arbitrarily small for $n_{0}$ and $n$ large enough with $n>n_{0}$.

$$
\begin{aligned}
& \mid \int_{\mathfrak{X}} \mu_{t}(d x) \int_{\tilde{a}_{\Lambda}^{m}(x)} d \lambda\left(x_{\Lambda}\right) P_{t}^{(\infty)}\left(x_{\Lambda} \mid a_{\Lambda}^{m}(x)\right) \tilde{F}\left(x_{\Lambda}\right) \\
& \quad-\int_{\mathfrak{X}} \mu_{t}(d x) \int_{\tilde{a}_{\Lambda}^{m}(x)} d \lambda\left(x_{\Lambda}\right) P_{t}^{n_{0}}\left(x_{\Lambda} \mid a_{\Lambda}^{m}(x)\right) \tilde{F}\left(x_{\Lambda}\right) \mid \\
& \mid \int_{\mathfrak{X}} \mu_{t}(d x) \int_{\hat{a}_{\Lambda}^{m}(x)} d \lambda\left(x_{\Lambda}\right) P_{t}^{n_{0}}\left(x_{\Lambda} \mid a_{\Lambda}^{m}(x)\right) \tilde{F}\left(x_{\Lambda}\right) \\
& \quad-\int_{\mathfrak{X}} \mu_{t}^{(n)}(d x) \int_{\tilde{a}_{\Lambda}^{m}(x)} d \lambda\left(x_{\Lambda}\right) P^{n_{0}}\left(x_{\Lambda} \mid a_{\Lambda}^{m}(x)\right) \tilde{F}\left(x_{\Lambda}\right) \mid \\
& \mid \int_{\mathfrak{X}} \mu_{t}^{(n)}(d x) \int_{a_{\Lambda}^{m}(x)} d \lambda\left(x_{\Lambda}\right) P_{t}^{n_{0}}\left(x_{\Lambda} \mid a_{\Lambda}^{m}(x)\right) \tilde{F}\left(x_{\Lambda}\right) \\
& \quad-\int_{\mathfrak{X}} \mu_{t}^{(n)}(d x) \int_{\tilde{a}_{\Lambda}^{m}(x)} d \lambda\left(x_{\Lambda}\right) P_{t}^{n}\left(x_{\Lambda} \mid a_{\Lambda}^{m}(x)\right) \tilde{F}\left(x_{\Lambda}\right) \mid .
\end{aligned}
$$


We start with estimating (3.37). Changing variables one obtain

$$
\begin{aligned}
(3.37) \leqq & \|F\|_{\infty} \int_{\mathfrak{x}_{A}^{m}\left(S_{t}^{(n)} x\right)} d \lambda\left(x_{A}\right) \\
& \cdot\left|P_{t}^{n_{0}}\left(x_{A} \mid a_{\Lambda}^{m}\left(S_{-t}^{(n)} x\right)\right)-P_{t}^{n}\left(x_{A} \mid a_{\Lambda}^{m}\left(S_{-t}^{(n)} x\right)\right)\right| .
\end{aligned}
$$

According to the Lemma 3.2 (see (3.15)),

$$
\begin{aligned}
& \mid P_{t}^{n_{0}}\left(x_{A} \mid a_{A}^{m}\left(S_{-t}^{(n)} x\right)\right)-P_{t}^{n}\left(x_{A}\left|a_{\Lambda}^{m}\left(\left(S_{-t}^{(n)} x\right)\right)\right|\right. \\
& \leqq\left(\exp \left[\gamma_{m}\left(n_{0} ;\left(S_{-t}^{(n)} x\right)\right)\right]-\exp \left[-\gamma_{m}\left(n_{0} ;\left(S_{-t}^{(n)} x\right)\right)\right]\right) \\
& P_{t}^{n_{0}}\left(x_{A} \mid a_{\Lambda}^{m}\left(S_{-t}^{(n)} x\right)\right) \\
& \leqq\left(\exp \left[\tilde{\gamma}_{m}\left(h_{0}, x\right)\right]-\exp \left[-\tilde{\gamma}_{m}\left(n_{0} ; x\right)\right]\right) \\
& P_{t}^{n_{0}}\left(x_{A} \mid a_{\Lambda}^{m}\left(S_{-t}^{(n)} x\right)\right) .
\end{aligned}
$$

Hence

$$
(3.37) \leqq\|F\|_{\infty} \int_{\mathfrak{X}} \mu(d x) \min \left[2, e^{\tilde{\gamma}_{m}\left(n_{0} ; x\right)}-e^{-\tilde{\gamma}_{m}\left(n_{0}, x\right)}\right] .
$$

By the use of the Lebesgue theorem, for any $\varepsilon>0$ we can find a sufficiently large $h_{0}$ such that (3.37) is smaller than $\varepsilon$ for all $n \geqq n_{0}$. The same arguments show that the term (3.35) vanishes as $n_{0} \rightarrow \infty$.

So (3.34) will be proven if we prove that for any fixed $n_{0},(3.36) \rightarrow 0$ as $n \rightarrow \infty$. Putting

$$
g(x)=\int_{\tilde{a}_{\Lambda}^{m}(x)} d \lambda\left(x_{\Lambda}\right) P_{t}^{n_{0}}\left(x_{\Lambda} \mid a_{\Lambda}^{m}(x)\right) \tilde{F}\left(x_{\Lambda}\right)
$$

it follows

$$
(3.36)=\left|\int_{\circledast} \mu(d x)\left[g\left(S_{-t} x\right)-g\left(S_{-t}^{(n)} x\right)\right]\right| .
$$

We shall prove that

$$
g\left(S_{-t} x\right)=\lim _{n \rightarrow \infty} g\left(S_{-t}^{(n)} x\right) \quad \text { for } \quad \mu \text {-a.a. } x \in \mathfrak{X}
$$

and this will imply that $(3.36) \rightarrow 0$ as $n \rightarrow \infty$ by the use of the bound $\|g\|_{\infty} \leqq\|F\|_{\infty}$ and Lebesgue theorem.

Let us fix $x \in \mathfrak{X}_{\varphi^{\prime}}$ such that $\mathscr{L}_{\varphi}\left(S_{-t} x\right) \notin \mathbb{N}$ where $\varphi^{\prime}(k)=1 \vee \log k$. Then

$$
\begin{aligned}
& \left|g\left(S_{-t} x\right)-g\left(S_{-t}^{(n)} x\right)\right| \leqq\|F\|_{\infty} \mid \int_{a_{\Lambda}^{m}\left(S_{-t} x\right)} d \lambda\left(x_{A}\right) P_{t}^{\left(n_{0}\right)}\left(x_{A} \mid a_{\Lambda}^{m}\left(S_{-t} x\right)\right) \\
& \left.\quad-\quad \int_{a_{\Lambda}^{m}\left(S_{-t}^{(n)} x\right)} d \lambda\left(x_{A}\right) P_{t}^{\left(n_{0}\right)}\left(x_{A}\right) a_{\Lambda}^{m}\left(S_{-t}^{(n)} x\right)\right) \mid \\
& =\left|\int_{a_{\Lambda}^{m}\left(S_{-t} x\right)} d \lambda\left(x_{A}\right)\left[P_{t}^{\left(n_{0}\right)}\left(x_{A} \mid a_{\Lambda}^{m}\left(S_{-t}^{(u)} x\right)\right)\right]\right|
\end{aligned}
$$

where the last equality in (3.43) holds if $n$ is sufficiently large, in virtue of Theorem 1) ii) and the definition of $\xi_{A}^{m}$.

Furthermore

$$
P_{t}^{n_{0}}\left(x_{A} \mid a_{\Lambda}^{m}\left(S_{-t}^{(n)} x\right)\right) \underset{n \rightarrow \infty}{\longrightarrow} P_{t}^{n_{0}}\left(x_{A} \mid a_{\Lambda}^{m}\left(S_{-t} x\right)\right) .
$$


(3.44) is consequence of the following convergence

$$
\exp \left[-h\left(S_{-t}^{\left(n_{0}\right)}\left(x_{A} \cup\left(S_{-t}^{(n)} x\right)_{\Lambda^{c}}\right)_{\Lambda_{n_{0^{+}}+}}\right)\right] \underset{n \rightarrow \infty}{\longrightarrow} \exp \left[-h\left(S_{-t}^{\left(n_{0}\right)}\left(x_{\Lambda} \cup\left(S_{-t} x\right)_{\Lambda^{c}}\right)_{\Lambda_{n_{0}+}+}\right)\right]
$$

due to Theorem 1 and the continuity of $h$ and of the Lebesgue theorem combined with the following estimate

$$
\begin{aligned}
& h\left(S_{-t}^{(n)}\left(x_{\Lambda} \cup\left(S_{-t}^{(n)} x\right)_{\Lambda^{c}}\right)_{A_{n_{0}+r}}\right) \geqq c H\left[\left(x_{A} \cup\left(S_{-t}^{(n)} x\right)_{\Lambda^{c}}\right)_{\Lambda_{n_{0}+r}}\right] \\
& \quad \geqq c \sum_{\left(p_{t}, q_{i}\right) \in x_{\Lambda}}\left\{p_{i}^{2} / 2+(k-2 v J) q_{i}^{2}+\lambda q_{i}^{4}\right\} \\
& \quad+\sum_{\left.\left(q_{j}, p_{j}\right) \in\left(S_{-t}^{(n) x}\right)\right)_{\Lambda_{0+r} \Lambda}}\left\{p_{j}^{2} / 2+(k-2 v J) q_{j}^{2}+\lambda q_{j}^{4}\right\}
\end{aligned}
$$

In fact $\exp [(3.46)]$ is the product of two terms one of which does not depend on $n$ and is integrable w.r.t. $d \lambda\left(x_{v}\right)$ and the other one is not depending on $x_{A}$ but converges as $n \rightarrow \infty$ and hence is bounded.

So (3.32) and hence Theorem 4 will be proven as consequence of the following statement:

$$
\mu_{t}\left(\left\{x \mid \mathscr{L}_{\varphi}(x)=m\right\}\right)=0 .
$$

For every $x \in \mathfrak{X}_{\varphi^{\prime}}$ then there exists a $j \in \mathbb{N}^{+}$such that

$$
\mathscr{L}_{\varphi}\left(S_{t} x\right)=\mathscr{L}^{(j)}\left(S_{t} x\right) / \varphi(j)=m .
$$

So (3.47) is implied by the fact that

$$
\mu_{t}\left(\left\{x \mid \mathscr{L}^{(j)}(x)=k, \quad k \in \mathbb{R}\right\}\right)=0 .
$$

Finally, (3.48) may be obtained by considering that the set in $\mathfrak{X}_{A}$ where $\mathscr{L}^{(j)}\left(x_{A}\right)$ take a fixed value has Lebesgue measure 0 , and using the locally absolute continuity of $\mu_{t}$ w.r.t. the Lebesgue measure. This final statement follows from the locally absolute continuity w.r.t. the Lebesgue measure of the approximating measures $\mu_{t}^{(h)}$.

\section{Appendix}

Proof of Theorem 1. By the use of the equation of the motion we obtain

$$
\frac{d}{d t} \mathscr{L}^{(i)}\left(S_{t}^{(n)} x\right)=J p_{i}^{(n)}(t, x)\left(\sum_{j \in v_{t}} q_{j}^{(n)}(t, x)\right) .
$$

The following estimates are obvious

$$
\left|p_{i}^{(n)}(t, x)\right| \leqq \sqrt{2 \mathscr{L}^{(i)}\left(S_{t}^{(n)} x\right)} ; \quad\left|q_{i}^{(n)}(t, x)\right| \leqq \sqrt{\frac{2}{k}} \sqrt{\mathscr{L}^{(i)}\left(S_{t}^{(n)} x\right)} .
$$

(A.1) and the hypothesis on $\varphi$ give the following integral inequality:

$$
\mathscr{L}_{\varphi}\left(S_{t}^{(n)} x\right) \leqq \mathscr{L}_{\varphi}(x)+\bar{a} \int_{0}^{t} \mathscr{L}_{\varphi}\left(S_{s}^{(n)} x\right) d s
$$

where $\bar{a}$ is a constant independent of $n$. 
Hence

$$
\mathscr{L}_{\varphi}\left(S_{t}^{(n)} x\right) \leqq e^{\bar{a}|t|}\left(\mathscr{L}_{\varphi}(x)\right) .
$$

Let us put:

$$
\begin{aligned}
u_{k}(t, x, n, m)= & \sup _{|i| \leqq k}\left\{\left|q_{i}^{(n)}(t, x)-q_{i}^{(n+m)}(t, x)\right|\right. \\
& \vee\left|p_{i}^{(n)}(t, x)-p_{i}^{(n+m)}(t, x)\right|
\end{aligned}
$$

Then by using the equation of the motion and in virtue of (A.2) and (A.4), there exists a constant $\bar{c}$ for which the following estimate holds:

$$
\begin{aligned}
u_{k}(t, x, n, m) \leqq & \int_{0}^{t} \sup _{|i| \leqq k}\left[\left|p_{i}^{(n)}(s)-p_{i}^{(n+m)}(s)\right|\right. \\
\vee & \mid K\left(q_{i}^{(n+m)}(s)-q_{i}^{(n)}(s)\right)+4 \lambda\left(q_{i}^{(n+m)}(s)-q_{i}^{(n)}(s)\right) \\
& \cdot\left(q_{i}^{(n+m)}(s)^{2}+q_{i}^{(n+m)}(s) q_{i}^{(n)}(s)+q_{i}^{(n)}(s)^{2}\right) \\
& \left.J \sum_{j \in v_{i}}\left|q_{j}^{(n)}(s)-q_{j}^{(n+m)}(s)\right|\right] d s \\
\leqq & \bar{c} e^{\bar{a} t} \varphi(k) \mathscr{L}_{\varphi}(x) \int_{0}^{t} u_{k+1}(s, x, n, m) d s .
\end{aligned}
$$

Interating the procedure $n-k$ times we obtain:

$$
u_{k}(t, x, n, m) \leqq \frac{\left(\bar{c} e^{\bar{a}|t|} \mathscr{L}_{\varphi}(x) \varphi(n) t\right)^{n-k} \bar{c} e^{\bar{a}|t|} \mathscr{L}_{\varphi}(x) \varphi(n)}{(n-k) !} .
$$

This is bound (2.14) with the first meaning of $(\bar{q}, \bar{p})$, that combined with (A.4) gives the assertion i). The estimates $\left(2.12^{\prime}\right),(2.14)$ with the second meaning of $(\bar{q}, \bar{p})$ and also (2.15), (2.16) may be obtained with the same arguments as above.

Now we prove ii). By i) one has:

$$
\lim _{n \rightarrow \infty} \mathscr{L}^{(i)}\left(S_{t}^{(n)} x\right)=\mathscr{L}^{(i)}\left(S_{t} x\right) \quad x \in \mathfrak{X}_{\varphi} .
$$

Fixed now $x \in \mathfrak{X}_{\varphi^{\prime}}$ it is enough to prove that there exists $b>0$ and $i_{n}$, such that $\left|i_{n}\right| \leqq b<+\infty$ for which

$$
\mathscr{L}_{\varphi}\left(S_{t}^{(n)} x\right)=\frac{\mathscr{L}^{\left(i_{n}\right)}\left(S_{t}^{n}(x)\right)}{\varphi\left(i_{n}\right)} .
$$

But the estimate:

$$
\frac{\mathscr{L}^{(i)}\left(S_{t}^{(n)} x\right)}{\varphi(i)} \leqq \mathscr{L}_{\varphi^{\prime}}(x) e^{\bar{a}_{3} t} \frac{\varphi^{\prime}(i)}{\varphi(i)}
$$

(where $\bar{a}_{3}$ depends only on $\varphi^{\prime}$ ) combined with the fact that $\varphi^{\prime}(k) / \varphi(k) \rightarrow 0$ gives (A.9) and hence proves ii).

Proof of Theorem 2. Theorem 2 is a consequence of the estimates in [13], Corollary 2.4. In fact, denoting $P_{\Lambda}\left(d x_{\Omega}\right), \Omega \subset \Lambda$, the probability distribution of $x_{\Omega}$ w.r.t. the measure

$$
\mu_{\Lambda}\left(d x_{\Lambda}\right)=\frac{\exp \left[-h\left(x_{\Lambda}\right)\right]}{\text { normalization factor }}
$$


one obtains the following estimates:

$$
P_{\Lambda}\left(d x_{\Omega}\right) \leqq A \exp \left[-k_{1} \sum_{i \in \Omega} p_{i}^{2}-k_{2} \sum_{i \in \Omega} q_{i}^{4}\right] d \lambda\left(x_{\Omega}\right)
$$

where $A, k_{1}, k_{2}$ are constant independent of $\Lambda$. Compactness arguments combined with (A.11) give the existence statement [14].

Still using estimate (A.11) one obtains :

$$
\int \mu(d x) e^{b \mathscr{L}((j))(x)}<M
$$

where $M$ is a constant not depending on $j$ and $b$ is sufficiently small. Finally by the Tchebyshev inequality:

$$
\mu\left(\left\{x \mid \mathscr{L}_{\varphi^{\prime}}(x)>\delta\right\}\right) \leqq A e^{-B \triangleleft}
$$

for some constant $A$ and $B$. This gives the thesis.

Acknowledgement. We thank S. Goldstein for having pointed us that an hypothesis was missing in the first version of this work. One of us (Yu.S.) is grateful to Italian National Council of Research for having supported his visit in Italy.

\section{References}

1. Lanford, C.E. III: Commun. Math. Phys. 9, 176 (1968); Commun. Math. Phys. 11, 257 (1969)

2. Dobrushin, R., Fritz, J.: Commun. Math. Phys. 55, 275 (1977); Commun. Math. Phys. 57, 67 (1977)

3. Lanford, O.E. III, Lebowitz, J.L., Lieb, E.H.: J. Stat. Phys. 16, 453 (1977)

4. Sinai, J.: Sov. Theor. Math. Phys. 12, 487 (1973); Vestn. Moscow Univ. Sez. I Math. Mech. 29, 152 (1974)

5. Lanford, O.E. III : Lecture notes in physics, Vol. 38. Moser, J. (ed.). Berlin, Heidelberg, New York: Springer 1975

6. Marchioro, C., Pellegrinotti, A., Presutti, E.: Commun. Math. Phys. 40, 175 (1975)

7. Presutti, E., Pulvirenti, M., Tirozzi, B.: Commun. Math. Phys. 47, 81 (1976)

8. Alexander, R.: Commun. Math. Phys. 49, 81 (1976)

9. Gurevich, B.M., Suhov, Yu.M.: Time evolution of Gibbs states. (In preparation) (1979)

10. Dobrushin, R.L., Suhov, Yu.M.: On the problem of the mathematical foundation of the Gibbs postulate in classical statistical mechanics. Proceedings of the $M \cap \Phi$ Conference, Rome (June 1977)

11. Ziman, J.M. : Principles of the theory of solids. New York: Cambridge University Press 1964

12. Rohlin, V.A.: Am. Math. Soc. Transl. 10, 1 (1962); Anosov, D.V.: Appendix in: Geodesic flows on closed Riemann manifolds with negative curvature. Proceedings of the Steklov Institute of Mathematics. Am. Math. Soc. n. 90 (1969)

13. Ruelle, D.: Commun. Math. Phys. 50, 189 (1976)

14. Dobrushin, R.: Theory Probab. Its Appl. XV 3, 458 (1970)

Communicated by J. L. Lebowitz

Received May 12, 1978; in revised form October 25, 1978 